Journal of Engineering and Applied Sciences 14 (2): 641-647, 2019

ISSN: 1816-949X

(C) Medwell Journals, 2019

\title{
Enhancement Underwater Image Using Histogram Equalization Based on Color Restoration
}

\author{
${ }^{1}$ Zahraa S. Abd-Al Ameer, ${ }^{1}$ Hazim G. Daway and ${ }^{2}$ Hana H. Kareem \\ ${ }^{1}$ Department of Physics, College of Science, \\ ${ }^{2}$ Department of Physics, College of Education, Al-Mustansiriyah University, Baghdad, Iraq
}

\begin{abstract}
In this study, the improvement of underwater images was re-correct color by design of a new method by using Histogram Equalization Based on Color Restoration (HEBCR). Proposed method adopted on applying traditimal histogram equalization on the image in RGB Model and then performed color correction by using (XYZ, LMS) color spaces and add to that apply logarithm mapping. Quantitative analyses indicate that the proposed method outperforms other methods as Multi Scale Retinex Algorithm with Color Restoration (MSRCR), Integrated Color Model (ICM), Unsupervised Color correction Method (UCM) and Rayleigh Stretching and Averaging Image Planes (RSAIP) by calculating no reference image quality assessments. Finally, we concluded from the results that the proposed HEBCR method is much better than the other methods for enhancement underwater images.
\end{abstract}

Key words: Underwater image processing, histogram equalization, image color restoration, UCM, RSAIP, HEBCR

\section{INTRODUCTION}

Underwater photograph processing is a challenging subject due to the physical properties of such an environment. Scientists accomplishing underwater research face the challenges of light absorption and diffusion consequences on underwater imaging (Baharudin et al., 2012; Gasparini and Schettini, 2003) as an example image captured deeper into the ocean turn greenish or bluish generally, the quality of underwater photographs is suffering from one of a factors, consisting of confined variety of visibility low evaluation, non-uniform lights, noise (Baharudin et al., 2012). As light travels in water, it rapidly loses intensity depending on the color spectrum wavelength, visible light absorbed at the longest wavelengths first (Schettini and Corchs, 2010). Red the maximum absorbed light color is decreased to $1 / 3$ of its depth after a depth of one $m$ and is largely lost after 4 and $5 \mathrm{~m}$ underwater (Hitam et al., 2013). In the meantime, the blue and violet lights are least absorbed compared with different wavelengths. The red color is the primary color absorbed through water at a depth of $5 \mathrm{~m}$, followed via. orange, yellow, green and blue. Underwater photos appear blue-green because those coloration components are least absorbed. There are methods to enhancement Images from them Color Model (ICM) (Iqbal et al., 2007, 2010), Unsupervised Color correction Method
(UCM), Rayleigh-Stretching and Averaging Image Planes Method (RSAIP) (Ghani and Isa, 2014) and Multi Scale Retinex (MSRCR) (Jobson et al., 1997a, b). These methods improve image contrast, increase image details and reduce noise.

\section{MATERIALS AND METHODS}

Image enhancement operations using proposed method histogram equalization applied on underwater images: Histogram equalization is utilized to enhancement the contrast of the photograph it spreads the intensity values over complete range histogram equalization technique can't be used for image tormented by irregular illumination in their backgrounds as this procedure best adds more pixels to the light areas of the image and eliminates more pixels from dark regions of the photo resulting in a high dynamic variety in the output photo. The purpose of histogram equalization is to spread out the contrast of a given image evenly in the course of the whole available dynamic range. In histogram equalization technique, it is the $\mathrm{R}(\mathrm{r})$ which denote probability density characteristic (pdf) of the lightness ranges in a given photo where the subscript is use for differentiating between the PDFs of the input and output photo. Assume that the following conversion performed on the input levels to obtain output (processed) intensity levels (Gonzalez and Woods, 2002):

Corresponding Author: Hazim G. Daway, Department of Physics, College of Science, Al-Mustansiriyah University, Baghdad, Iraq 


$$
\mathrm{s}=\mathrm{T}(\mathrm{s})=\int_{0}^{\mathrm{r}} \operatorname{Pr}(\mathrm{w}) \mathrm{dw}
$$

where, $w$ is a dummy variable of integration that the probability density function of the output levels is uniform that is (Gonzalez and Woods, 2002):

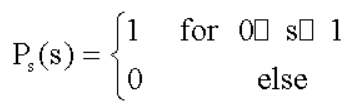

When dealing with discrete quantities, we research with histograms and call the preceding technique histogram equalization where (Tao and Asari, 2004):

$$
\begin{aligned}
& \mathrm{s}_{\mathrm{k}}=\sum_{\mathrm{j}=0}^{\mathrm{k}} \mathrm{p}_{\mathrm{r}}\left(\mathrm{r}_{\mathrm{j}}\right)=\sum_{\mathrm{j}=0}^{\mathrm{k}} \frac{\mathrm{n}_{\mathrm{j}}}{\mathrm{N}} \\
& \mathrm{k}=0,1,2, \ldots, \mathrm{L}, \mathrm{L}=255
\end{aligned}
$$

for lightness band with 8 bit/pixel, $\mathrm{s}_{\mathrm{k}}$ corresponding normalized intensity level of the output image and $n_{j}$ being the number of pixel with intensity level $\mathrm{j}$ and $\mathrm{n}$ is the total number. Equation 3 is represent the Cumulative Probability Density Function (CPDF) $r_{j}$ is normalized intensity level of the input image corresponding to the (un-normalized) intensity level this algorithm summarized by using following steps:

\section{Algorithm 1:}

1. Input color image $\mathrm{C}_{\mathrm{i}}, \mathrm{i}=1,2,3$ (red, green, blue) components

2. Normalize each component $\mathrm{r}_{\mathrm{j}}(\mathrm{i})=\mathrm{C}_{\mathrm{i}} / 255$ and calculated frequency of occurrence each gradual level

3. Compute histogram from $p\left(r_{j}(i)\right)=n_{j}(i) / N$ where, $N$ being the size of image

4. Calculate cumulative histogram by:

$$
\mathrm{s}_{\mathrm{k}}(\mathrm{i})=\sum_{\mathrm{j}=0}^{\mathrm{k}} \frac{\mathrm{n}_{\mathrm{j}}(\mathrm{i})}{\mathrm{N}}
$$

where, $\mathrm{k}=0,1, \ldots, 255$

5. Replace each normalized component $r_{j}(i)$ by value of $s_{k}(i)$, we get the output image

Image color restoration: Recovering a minimum realistic color of underwater scenes is a totally hard within the beginning photograph conversion from RGB to $\mathrm{XYZ}$ space, the conversion is given by using Eq. 5:

$$
\begin{array}{rrr}
0.5141 & 0.3239 & 0.1604 \\
\mathrm{M}_{\text {RGB to XYZ }}=0.2651 & 0.6702 & 0.0641 \\
0.0241 & 0.1228 & 0.8444
\end{array}
$$

After that complete transforming color image from basic XYZ color space to LMS color space, the transform is given by Eq. 6:

$$
\begin{array}{rrr}
0.3897 & 0.6890 & 0.0787 \\
\mathrm{M}_{\mathrm{XYZ} \text { to LMS }}=-0.2298 & 1.1834 & 0.0464 \\
0.0000 & 0.0000 & 1.0000
\end{array}
$$

finally, apply logarithm model to color restoration by using Eq. 7:

$$
I_{(x, y, a, b)}^{\prime}=b \log \left[1+a \frac{I_{n}(x, y)}{\sum_{n=1}^{3} I_{n}(x, y)}\right]
$$

where, $b=100, a=125$. Finally, note great improvement in the image.

Quantitative analysis: Quantitative analysis is included in the study to evaluate the resultant image. The quantitative evaluation is only used to support the visual observation. In this study, the quantitative evaluation is done based on: entropy average gradient and Natural Image Quality Evaluator (NIQE).

Entropy value: Entropy can be defined as the abundance of image information which measures the image information content which is interpreted as the average uncertainty of information source. In an image, entropy is defined as corresponding states of intensity level which individual pixels can adopt. It is calculated as the summation of the products of the probability of outcome multiplied by the $\log$ of the inverse of the outcome probability (Ye, 2009):

$$
\mathrm{H}_{(\mathrm{x})}=\sum_{\mathrm{x}=0}^{\mathrm{L}} \mathrm{p}_{(\mathrm{x})} \log _{2} \mathrm{p}_{(\mathrm{x})}
$$

Average gradient: Average gradient shows the fine contrast, texture characteristic and clarity of an image. The above value of average gradient indicates that the image has more intensity levels and is clearer. Wu et al. (2005), the average gradient can be defined as follow:

$$
\nabla \bar{G}=\frac{1}{(M-1)(N-1)} \sum_{i=1}^{M-1} \sum_{j=1}^{N-1} \sqrt{\frac{\nabla_{i}^{2} f(i, j)+\nabla_{j}^{2} f(i, j)}{2}}
$$

Where:

$$
\begin{array}{ll}
\nabla_{\mathrm{i}}^{2} \mathrm{f}(\mathrm{i}, \mathrm{j}) \text { and }= & \text { The gradients on the row and } \\
\nabla_{\mathrm{j}}^{2} \mathrm{f}(\mathrm{i}, \mathrm{j}) & \text { column direction, respectively } \\
\mathrm{M} \text { and } \mathrm{N} & =\text { The numbers of the row and column of } \\
& \text { the enhanced image, respectively }
\end{array}
$$

Natural Image Quality Evaluator (NIQE): Natural Image Quality Evaluator (NIQE) is some other No-Reference 
photograph Quality Assessment (NR-IQA) Version that best makes use of measurable deviations from statistical regularities discovered in natural photograph without education on human-rated distorted photographs NIQE assesses picture great without information of expected distortions or human reviews of them. NIQE is based on the construction of a quality aware' collection of statistical features based on a simple and successful space domain Natural Scene Statistic (NSS) Model. These features are derived from a corpus of natural, undistorted images. NIQE is applied by computing the 36 identical features patches of the same size $p \times p$ from the image to be quality analyzed, fitting them with the Multivariate Gaussian (MVG) Model, then comparing its MVG fit to the natural MVG Model. In the implementation of the NIQE, the patch size was set $96 \times 96$ to as implemented by the researchers in their researches. However, the stable performance across patch sizes ranging from $32 \times 32$ to $160 \times 160$. Thus, the quality of an image, $D$ is expressed as the distance between the quality aware NSS feature model and the MVG fit to the features extracted from the distorted image:

$$
D=\sqrt{\left(\left(v_{1}-v_{2}\right)^{T}\left(\frac{\sum_{1}+\sum_{2}}{2}\right)^{-1}\left(v_{1}-v_{2}\right)\right)}
$$

where, $v_{1} v_{2}$ and are the mean vectors and covariance matrices of the natural MVG Model and the distorted image's MVG Model, respectively. The low value of NIQE demonstrates the better image quality.

\section{RESULTS AND DISCUSSION}

The 5 methods have been implemented and tested (ICM, UCM, RSAIP, MSRCR and the proposed method). Simulation experiments have been conducted on 5 popular test images. By using MATLAB Software program and to simulate the proposed method and association of different methods together, we get some result which help as to find the advantages of these methods and techniques. Table 1 shows the comparative values of entropy, average gradient and NIQE for the images shown in Fig. 1-8. As can be seen in Fig. 4, the quantitative performance of the proposed method stands out among the other methods in term of entropy.

In Fig. 5, the proposed method stands out among the other methods in terms of entropy, average gradient and NIQE. In Fig. 6, the proposed method stands out among the other methods in terms of entropy, average gradient. In Fig. 7, the proposed method stands out among the other methods in term NIQE. In Fig. 8, the proposed
Table 1: Quantitative results of entropy, NIQE and average gradient

\begin{tabular}{lrrr}
\hline Images/Methods & Entropy & Average gradient & NQE \\
\hline Figure 4 & & & \\
ICM & 7.2553 & 9.9017 & 7.5386 \\
UCM & 7.2553 & 9.9017 & 7.5386 \\
RSAIP & 6.4796 & 8.9280 & 7.7465 \\
MSRCR & 7.0834 & 15.7724 & 7.2803 \\
Proposed method & 7.2980 & 11.7783 & 7.4265 \\
Figure 5 & & & \\
ICM & 6.9460 & 9.8518 & 5.6543 \\
UCM & 6.9460 & 9.8518 & 5.6543 \\
RSAIP & 6.0859 & 11.6049 & 5.5848 \\
MSRCR & 5.8149 & 8.9711 & 5.8479 \\
Proposed method & 7.3442 & 18.0087 & 4.3413 \\
Figure 6 & & & \\
ICM & 7.0418 & 19.2810 & 3.6091 \\
UCM & 7.0418 & 19.2810 & 3.6091 \\
RSAIP & 6.2906 & 19.6536 & 3.0090 \\
MSRCR & 6.1431 & 16.0146 & 3.5940 \\
Proposed method & 7.3625 & 30.5290 & 3.2454 \\
Figure 7 & & & \\
ICM & 7.3955 & 16.8531 & 3.3281 \\
UCM & 7.3955 & 16.8531 & 3.3281 \\
RSAIP & 7.1341 & 17.4385 & 3.6454 \\
MSRCR & 7.1923 & 22.7195 & 2.9715 \\
Proposed method & 7.1215 & 19.4968 & 2.8209 \\
Figure 8 & & & \\
ICM & 6.7953 & 9.6761 & 8.2226 \\
UCM & 6.7953 & 9.6761 & 8.2226 \\
RSAIP & 5.6262 & 8.0293 & 7.8625 \\
MSRCR & 6.8186 & 17.5389 & 6.3686 \\
Proposed method & 7.3666 & 14.3671 & 7.0059 \\
\hline
\end{tabular}

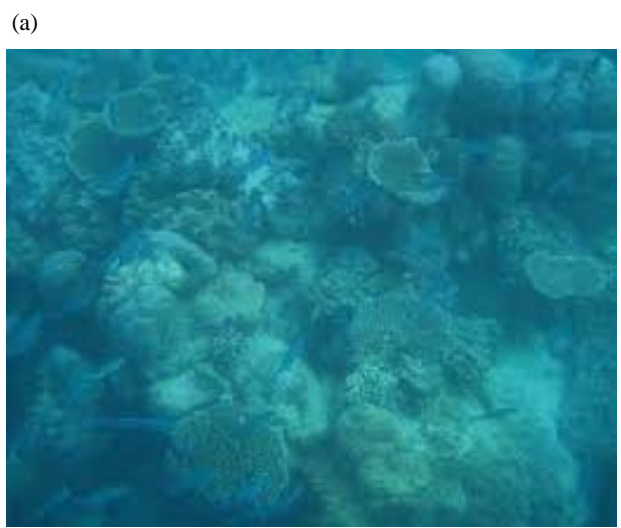

(b)

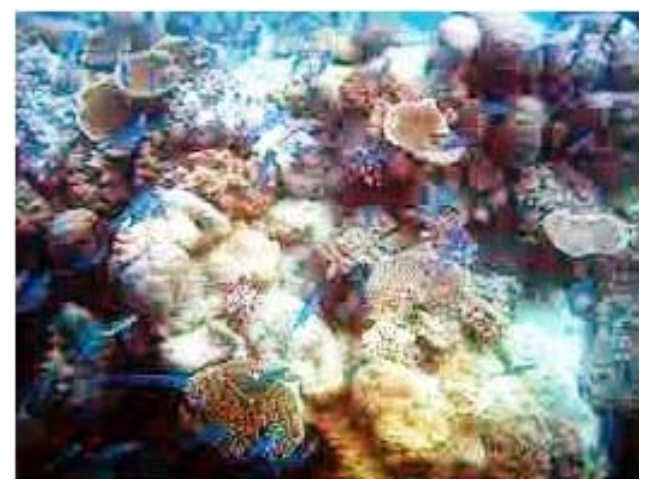

Fig. 1: Applied histogram equalization on the image: a) Original image and b) Applied (HE) 
(a)

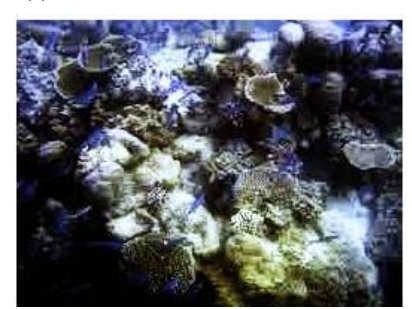

(b)

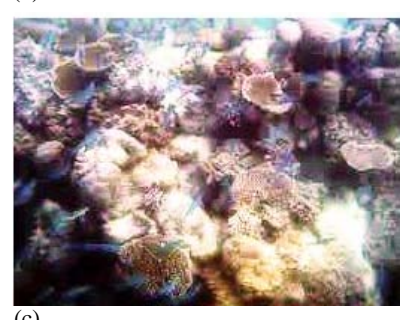

(c)

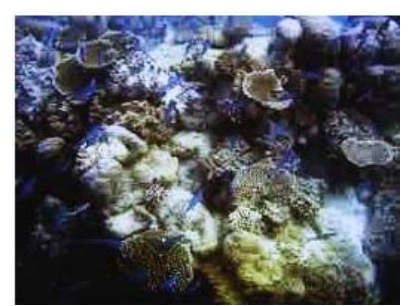

Fig. 2: a) Transform to XYZ; b) Transform to LMS and c) Applied logarithm model method stands out among the other methods in term entropy. the proposed method has been proven as a suitable technique to be used for enhancement of underwater images.

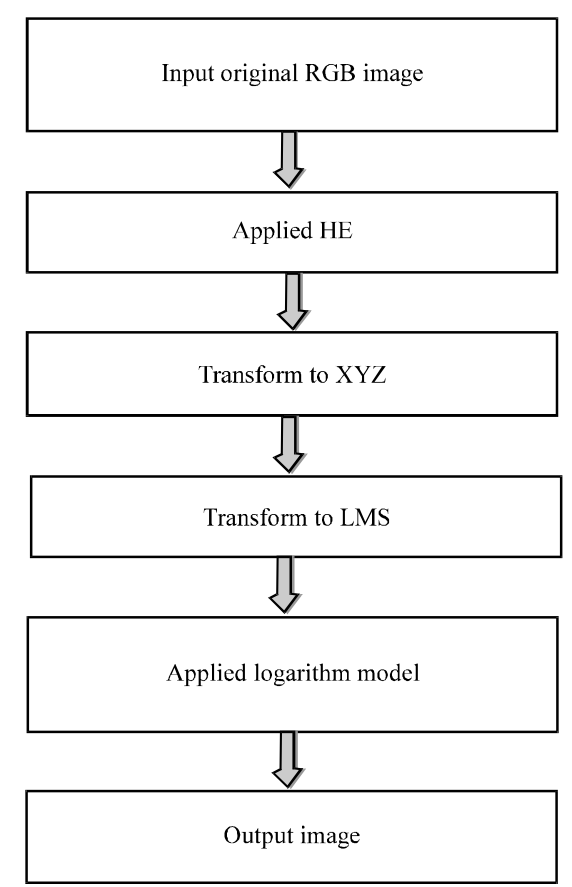

Fig. 3: Flowchart of the proposed method (a)

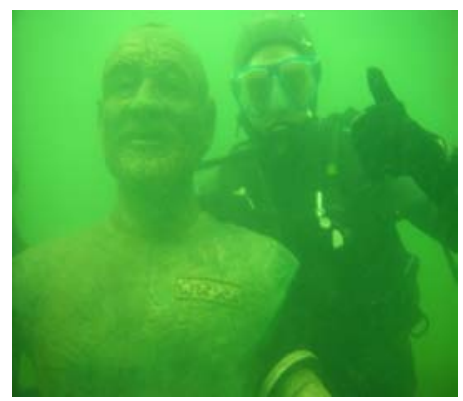

(d)

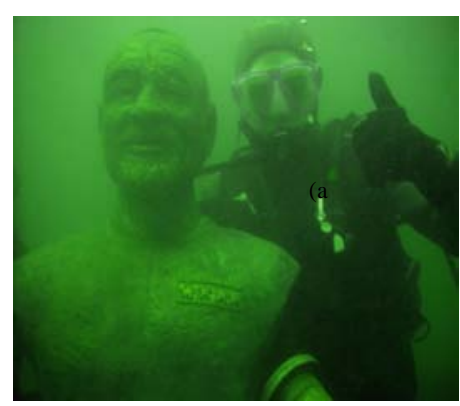

(b)

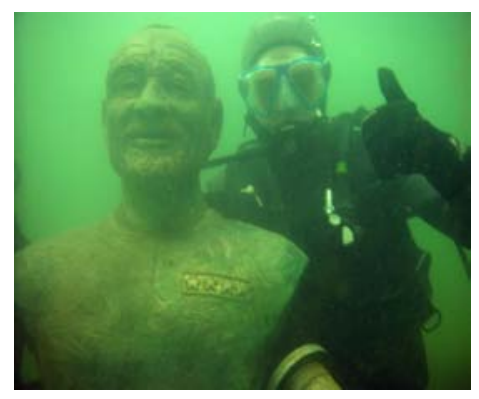

(e)

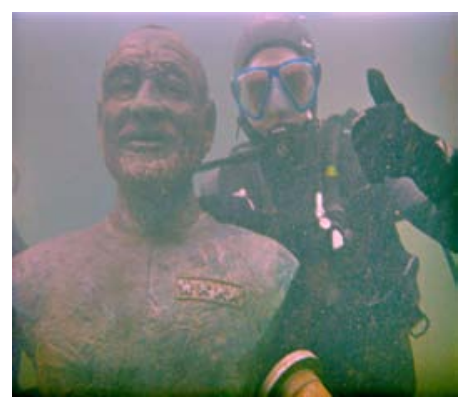

(c)

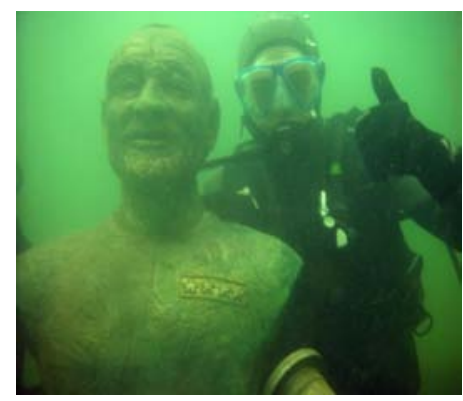

(f)

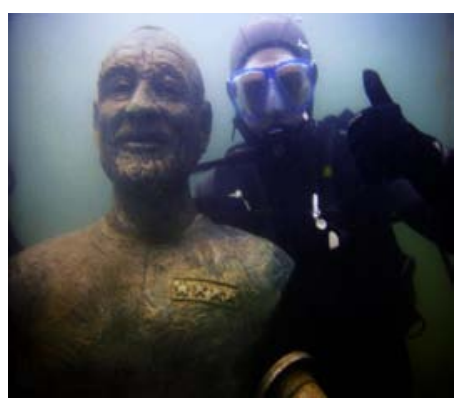

Fig. 4: a) Original image; b) ICM; c) UCM; d) RSAIP; e) MSRCR and f) Proposed method 
(a)

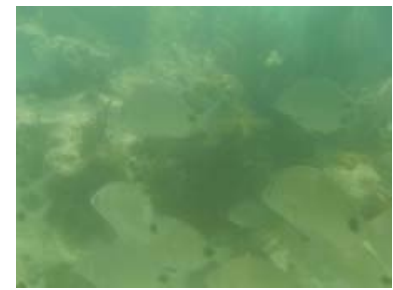

(d)

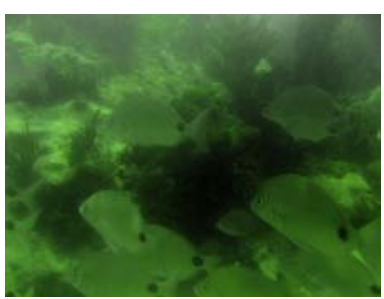

(b)

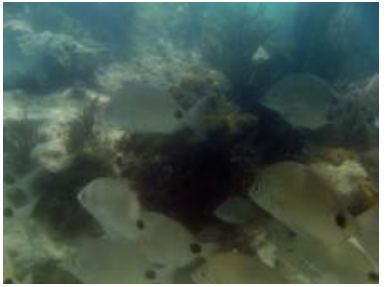

(e)

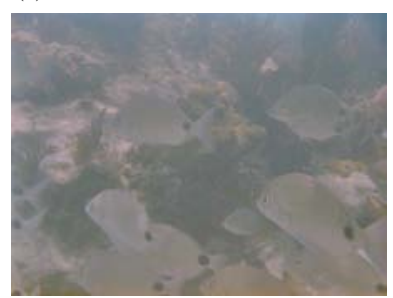

(c)

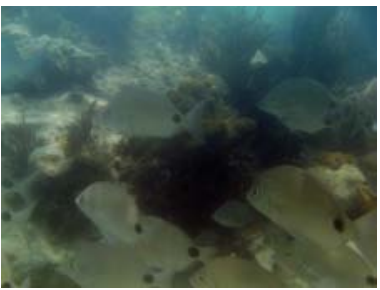

(f)

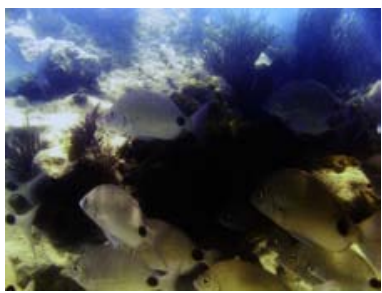

Fig. 5: a) Original image; b) ICM; c) UCM; d) RSAIP; e) MSRCR and f) Proposed method

(a)

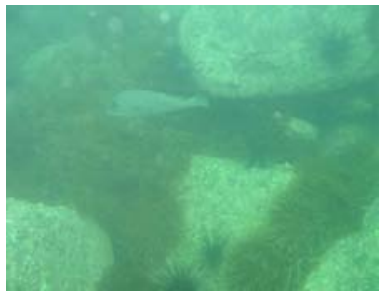

(d)

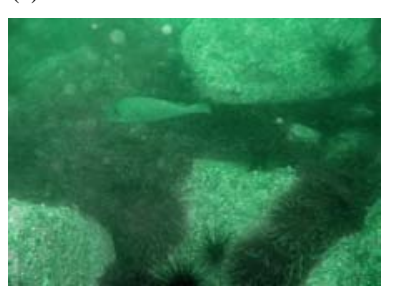

(b)

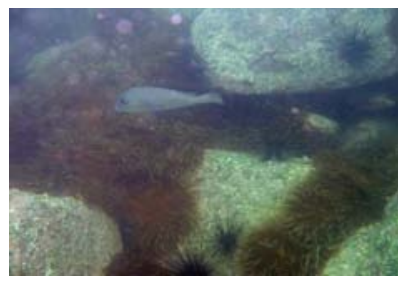

(e)

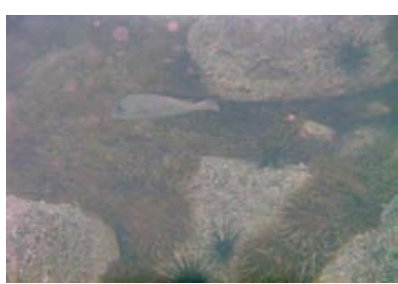

(c)

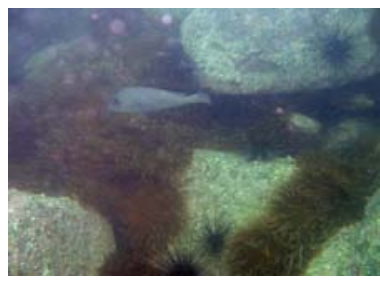

(f)

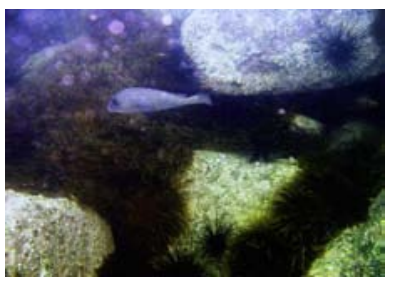

Fig. 6: a) Original image; b) ICM; c) UCM; d) RSAIP; e) MSRCR and f) Proposed method

(a)

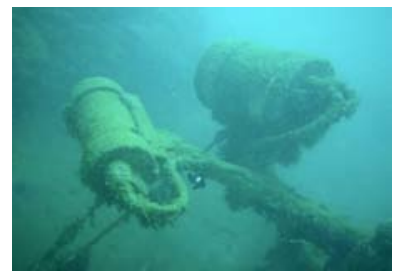

(d)

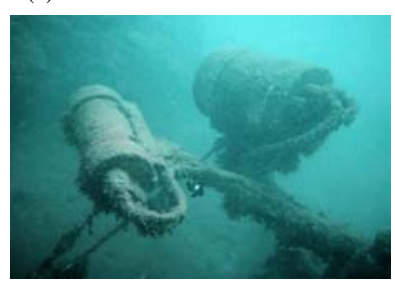

(b)

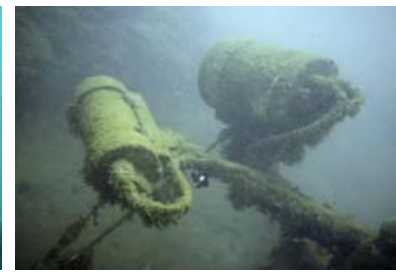

(e)

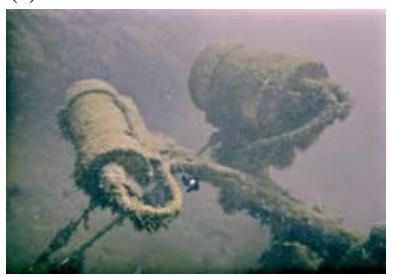

(c)

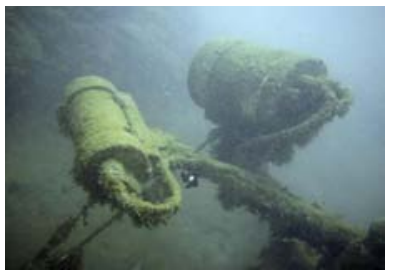

(f)

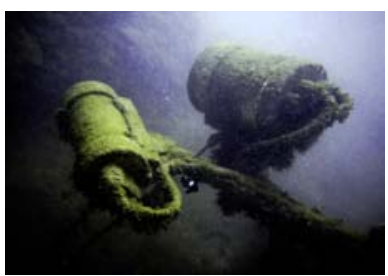

Fig. 7: a) Original image; b) ICM; c) UCM; d) RSAIP; e) MSRCR and f) Proposed method 
(a)

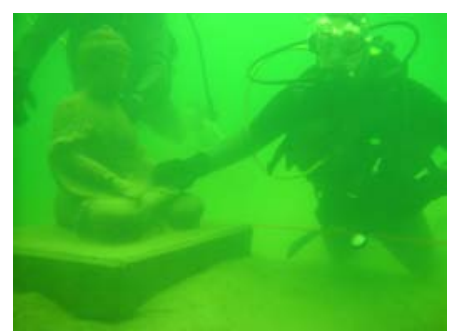

(d)

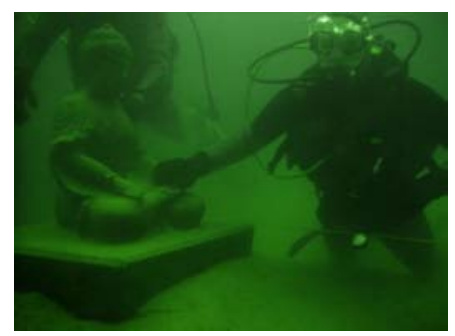

(b)

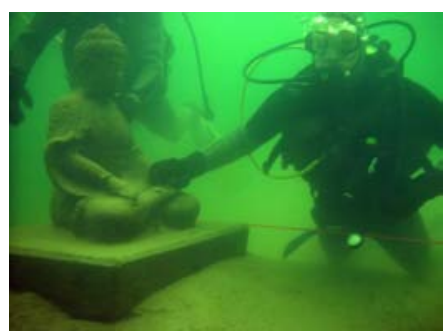

(e)

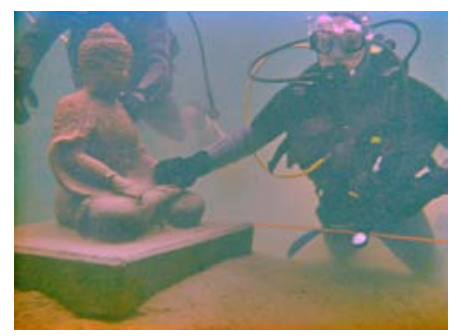

(c)

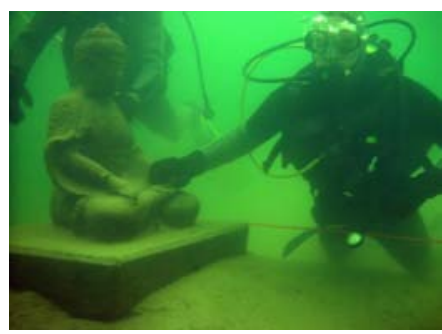

(f)

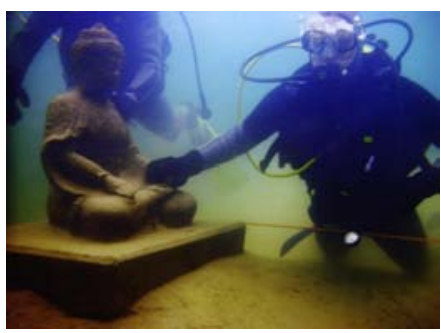

Fig. 8: a) Original image; b) ICM; c) UCM; d) RSAIP; e) MSRCR and f) Proposed method

\section{CONCLUSION}

The proposed algorithm characterized by the flexibility, simplicity and it succeeded in retrieving the missing details of by processing of color components. While the MSRCR algorithm did not improve the colors well. Also, ICM, UCM and RSAIP algorithms didn't success to improve the lightening of the color images. The proposed method was enabled to improve both lightness and contrast in the poor visibility underwater images, provides better visual representation comparing to the other methods (ICM, UCM, RSAIP and MSRCR).

\section{REFERENCES}

Baharudin, B.B., M. Kushairi and M. Rajuddin, 2012. Significance level of image enhancement techniques for underwater images. Proceedings of the 2012 International Conference on Computer \& Information Science (ICCIS) Vol. 1, June 12-14, 2012, IEEE, Kuala Lumpeu, Malaysia, ISBN:978-1-4673-1937-9, pp: 490-494.

Gasparini, F. and R. Schettini, 2003. Color correction for digital photographs. Proceedings of the 12th International Conference on Image Analysis and Processing (ICIAP 2003), September 17-19, 2003, IEEE, Mantova, Italy, ISBN:0-7695-1948-2, pp: 646-651.

Ghani, A.S.A. and N.A.M. Isa, 2014. Underwater image quality enhancement through Rayleigh-stretching and averaging image planes. Intl. J. Nav. Archit. Ocean Eng., 6: 840-866.
Gonzalez, R.C. and R.E. Woods, 2002. Digital Image Processing. Pearson Education Inc., Upper Saddle River, NJ., USA.

Hitam, M.S., E.A. Awalludin, W.N.J.H.W. Yussof and Z. Bachok, 2013. Mixture contrast limited adaptive histogram equalization for underwater image enhancement. Proceedings of the 2013 International Conference on Computer Applications Technology (ICCAT), January 20-22, 2013, EEE, Sousse, Tunisia, ISBN:978-1-4673-5284-0, pp: $1-5$.

Iqbal, K., M.O. Odetayo, A.E. James, R.A. Salam and A.Z. Talib, 2010. Enhancing the low quality images using Unsupervised Colour Correction Method. Proceedings of the 2010 IEEE International Conference on Systems, Man and Cybernetics, October 10-13, 2010, IEEE, Istanbul, Turkey, ISBN:978-1-4244-6586-6, pp: 1703-1709.

Iqbal, K., R.A. Salam, A. Osman and A.Z. Talib, 2007. Underwater image enhancement using an integrated colour model. Int. J. Comput. Sci., 34: 529-534.

Jobson, D., Z. Rahman and G.A.Woodell, 1997b. A multiscale retinex for bridging the gap between color images and the human observation of scenes. IEEE Trans. Image Proc., 6: 965-976.

Jobson, D.J., Z. Rahman and G.A. Woodell, 1997a. Properties and performance of a center/surround retinex. IEEE Trans. Image Proc., 6: 451-462. 
Schettini, R. and S. Corchs, 2010. Underwater image processing: State of the art of restoration and image enhancement methods. EURASIP J. Adv. Signal Process., 2010: 1-14.

Tao, L. and V. Asari, 2004. An integrated neighborhood dependent approach for nonlinear enhancement of color images. Proceedings of the International Conference on Information Technology: Coding and Computing (ITCC 2004) Vol. 02, April 5-7, 2004, IEEE, Las Vegas, USA, ISBN:0-7695-2108-8, pp: 138-139.
Wu, J., H. Huang, Y. Qiu, H. Wu and J. Tian et al., 2005. Remote sensing image fusion based on average gradient of wavelet transform. Proceedings of the IEEE International Conference Mechatronics and Automation Vol. 4, July 29-August 1, 2005, IEEE, Niagara Falls, Canada, ISBN:0-7803-9044-X, pp: 1817-1821.

Ye, Z., 2009. Objective assessment of nonlinear segmentation approaches to gray level underwater images. Intl. J. Graphics, Vision, Image Process., 9: $39-46$. 